\title{
Road Assessment Model and Pilot Application in China
}

\author{
Tiejun Zhang, ${ }^{1}$ Chengcheng Tang, ${ }^{1}$ Greg Smith, ${ }^{2}$ and Lingtao $\mathrm{Wu}^{1}$ \\ ${ }^{1}$ Research Institute of Highway, Ministry of Transport, Beijing, China \\ ${ }^{2}$ International Road Assessment Programme, London, UK \\ Correspondence should be addressed to Tiejun Zhang; jn.wan@rioh.cn \\ Received 6 March 2014; Revised 23 April 2014; Accepted 23 April 2014; Published 14 May 2014 \\ Academic Editor: Wuhong Wang
}

Copyright (C) 2014 Tiejun Zhang et al. This is an open access article distributed under the Creative Commons Attribution License, which permits unrestricted use, distribution, and reproduction in any medium, provided the original work is properly cited.

\begin{abstract}
Risk assessment of roads is an effective approach for road agencies to determine safety improvement investments. It can increases the cost-effective returns in crash and injury reductions. To get a powerful Chinese risk assessment model, Research Institute of Highway (RIOH) is developing China Road Assessment Programme (ChinaRAP) model to show the traffic crashes in China in partnership with International Road Assessment Programme (iRAP). The ChinaRAP model is based upon RIOH's achievements and iRAP models. This paper documents part of ChinaRAP's research work, mainly including the RIOH model and its pilot application in a province in China.
\end{abstract}

\section{Introduction}

With the fast urbanization and motorization in China, the highway network is expanding quickly. By the end of 2011, the total length of operating technical highways is more than 35.37 million $\mathrm{km}$ including 85,000 km expressways [1]. However, China is also facing huge traffic safety challenge as a result of blasted traffic volume and complex traffic compositions. In 2012, about 60,000 persons lost their lives in traffic accidents in China [2]. The accidents have also caused huge economic loss.

Since traffic safety issue is a world disaster, many research and actual countermeasures have been performed to improve traffic safety conditions. In current traffic safety theory system, traffic safety management and traffic safety risk assessment [3] are two main systematic tools to direct actual traffic safety projects. Among these two tools, traffic safety risk assessment has been shown to be an effective method to evaluate the highway network safety level. It also makes economic plan based on benefit to cost $(B / C)$ analysis, which is the ratio of the estimated safety benefit to the cost of actual countermeasures. Traffic safety risk assessment is an integration of historical effective achievements including accident prediction model, countermeasure effect appraisal, and economy analysis. Many methods have been used to predict the relationship between accidents and roadway geometric characters as well as traffic related explanatory factors, such as multiple linear regression, Poisson regression and negative binomial regression, the zero-inflated Poisson model, and the generalized estimating equations [4-9]. To make decisions on appropriate treatments selection, know the estimated effects of such countermeasures and support the address of crash problem based on the expected crash reduction. Many safety countermeasure effect appraisals are performed including empirical and statistic (such as EB model) methods and the results eventually are expressed as crash reduction factors [10-13]. The main objective of economy analysis is to express the crash loss in economic index which can match the cost and benefit very well [14].

iRAP model has been undertaken in many countries all over the world as a sophisticated risk assessment method. The iRAP method includes data collection, survey, coding, assessment, and economic appraisal phrases [4]. The building of the "China Road Assessment Program (ChinaRAP)" project was undertaken in partnership between the RIOH and iRAP, and the objective was to develop road infrastructure risk assessment models that draw on iRAP and RIOH knowledge for application in China. During the pilot project, both $\mathrm{RIOH}$ and iRAP risk assessment models were used, and this paper introduces the RIOH method in the research. This paper shows the basic framework of RIOH model, its pilot application procedure, and the assessment results. 
TABLE 1: RIOH standard of safety levels.

\begin{tabular}{lc}
\hline Level & Standard scope (injury accidents per $\mathrm{km}$ ) \\
\hline 5 & 0.00 to 0.24 \\
4 & 0.24 to 0.35 \\
3 & 0.35 to 0.51 \\
2 & 0.51 to 0.85 \\
1 & $>0.85$ \\
\hline
\end{tabular}

\section{RIOH Risk Assessment Model}

The $\mathrm{RIOH}$ risk assessment model is an integration of research achievements by RIOH, including safety audit theory, accident predication model, countermeasure effect appraisal, and accident loss economy analysis.

$\mathrm{RIOH}$ risk scores are indicated by the injury accident rate calculated by basic accident rate and the influence of actual traffic safety factors. The values of the influence of actual traffic safety factors are generated from statistical models and some before-and-after analysis results. To express the safety condition, 5 levels are used in which 1 is the worst and 5 is the best, as shown in Table 1. In the safety level map, green is the lowest risk and black is the highest risk. Based on the analysis of risk scores, the economic analysis provides some estimation of the benefit of countermeasures and $B / C$ rate.

In $\mathrm{RIOH}$ model, highways are separated into general segments, village segments, and intersections. To show the framework of RIOH model, an example for all accidents calculation of general segments is illustrated below. It includes a basic safety condition (showed in Table 2 for general segments) and safety index of actual factors:

$$
P_{c q}=\operatorname{expo} \times B P_{c q} \times P_{c q r}=\operatorname{expo} \times B P_{c q} \times P_{c q 1} \cdots \times P_{c q m},
$$

where $P_{c q}$ is all accidents rate of general segment, expo = $(365 \times z r \times y) / 1000000, z r$ is traffic volume, $y$ is year, $B P_{c q}$ is basic accident rate of $P_{c q}, P_{c q r}$ is actual accident rate of $P_{c q}$, and $P_{c q m}$ is the actual $m$ th factor of $P_{c q}$, such as for lighting. For total accidents of general segments, $B P_{c q}=0.69$.

The basic road condition of general segments is shown in Table 2.

\section{Model Application on Road Network}

3.1. Road Network. In total, $374.1 \mathrm{~km}$ of 9 roads in China was surveyed and fully assessed for the pilot project. Table 3 summarizes the length of class of each road. For the purpose of this study, the informal name of "class $2 a$ " is given to roads that are typically class 2 but have been upgraded to have two lanes of traffic in each direction and do not have median separation.

3.2. Road Survey Data. To match the basic data requirement of assessment, data collection and survey work was performed on the road network. Data collection included traffic volume, typical countermeasure costs, and economics and demographics data. Road network was surveyed using RIOH's existing survey method, including two video cameras,
TABLE 2: Basic road condition of general segments.

\begin{tabular}{lc}
\hline Attributes & Description \\
\hline Design speed & Low $(\leq 30 \mathrm{~km} / \mathrm{h})$ \\
Segment character & Not bridge or tunnel \\
Surface character & Pitch surface \\
Directing signs & No \\
Warning signs & No \\
Lighting & No \\
Medial marking & No \\
Edge marking & No \\
Pedestrian crossing line & No \\
Linked to village & No \\
Turing angle & Straight \\
Gradient & Plain \\
Bicycle percentage & Average $(3 \%)$ \\
Bus percentage & Average $(10 \%)$ \\
Motorcycle percentage & Average $(7 \%)$ \\
Driveway density & No \\
\hline
\end{tabular}

GPS, and recording of observations by road engineers. For using the survey videos and GPS, road attributes on building ChinaRAP network were coded at $0.1 \mathrm{~km}$ intervals. A summary of the road attributes by road class is shown in Table 4.

\section{Pilot Application Procedure and Results}

Based on the coding data and some other basic data such as countermeasure cost, the risk assessment is performed. The actual application procedure includes 3 stages: model assessment to produce risk maps, investment plan analysis to order the countermeasures by $B / C$ rate, and the predictive effect analysis to direct the location where we will get best accident reduction.

4.1. Model Assessment Results. Model assessment results include two parts; one is total assessment results and the second is results for every segment to produce the risk map. Table 5 shows the predicted accident density on pilot road network. It is noted that although 17 attributes were used to set the baseline accident rate for general segments (as shown in Table 4) and a total of 18 attributes were used for all segment types (common, village, and intersection), for the purposes of this pilot application, a total of ten attributes were used.

The predictive accident density of all users is showed in Table 6, whose total predictive injury accident numbers are 226.6 injury accidents. The actual injury accident number with accurate location in the network is about 153.4 per year (accident density is 0.41 per $\mathrm{km}$ ). However, as there are only $63 \%$ accidents which have detailed location information, the total accident number is actually about 242.3 per year, which shows that $\mathrm{RIOH}$ results underestimate the level of risk on the roads.

Table 6 shows the predicted accident densities according to the thresholds discussed earlier (as shown in Table 12).

Figure 1 shows the accident densities in map form. 
TABLE 3: Network surveyed.

\begin{tabular}{|c|c|c|c|c|c|c|}
\hline Road name & Class $1(\mathrm{~km})$ & Class $2 \mathrm{a}(\mathrm{km})$ & Class $2(\mathrm{~km})$ & Class $3(\mathrm{~km})$ & Urban $(\mathrm{km})$ & Total $(\mathrm{km})$ \\
\hline Road 2 & 36.3 & 5.2 & 4.7 & & & 46.2 \\
\hline Road 9 & & & 31.1 & & & 31.1 \\
\hline Road 3 & 11.6 & & 32.1 & & & 43.7 \\
\hline Road 4 & & & 44.3 & & & 44.3 \\
\hline Road 1 & & 1.0 & 58.0 & & & 59.0 \\
\hline Road 5 & & & 6.0 & & 13.6 & 19.6 \\
\hline Road 6 & 7.2 & 14.2 & 31.4 & & & 52.8 \\
\hline Road 7 & & & 27.8 & & & 27.8 \\
\hline Road 8 & & & & 49.6 & & 49.6 \\
\hline Total & 55.1 & 20.4 & 235.4 & 49.6 & 13.6 & 374.1 \\
\hline
\end{tabular}

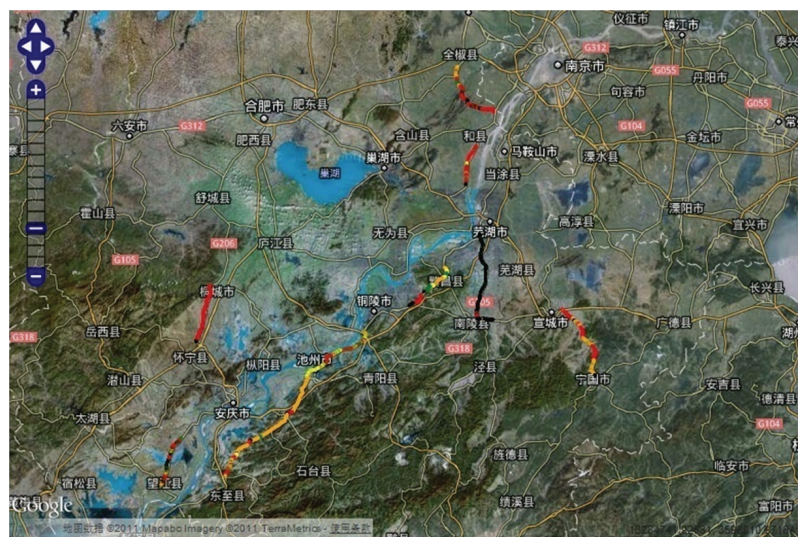

FIGURE 1: RIOH all occupants of accident density map.

4.2. Investment Plan. Economy analysis is based on the calculation of life cost and countermeasure cost together with the difference of risk score before-and-after countermeasures are implemented, which can measure economic invest and accident loss in the same way. To match the model skeleton and express it simply, the safety effect of countermeasures was calculated individually, and no relation is thought for complex countermeasures. For the purposes of this pilot, six kinds of countermeasures were appraised, as shown in Table 7.

Table 8 shows a summary of the investment plan results, in which center marking, pedestrian crosswalk, and roadside marking are with the highest benefit cost ratios. Table 8 indicates that an investment of approximately RMB 130,246,350 could prevent 86.34 serious accidents per year in the 10 -year future. Over 10 years, more than RMB 130,246,350 in injury crash costs could be saved, leading to a benefit cost ratio of $15.96: 1$.

4.3. Predictive Results. It is important to pinpoint the locations where we can get the biggest accident reduction effects in actual improvement projects. Figure 2 map shows the locations where injury accidents rate (per km per year for all users) could be prevented over the next 10 years if the investment plan is implemented. Levels 1 and 2 sections of road are where the most injury accidents could be prevented

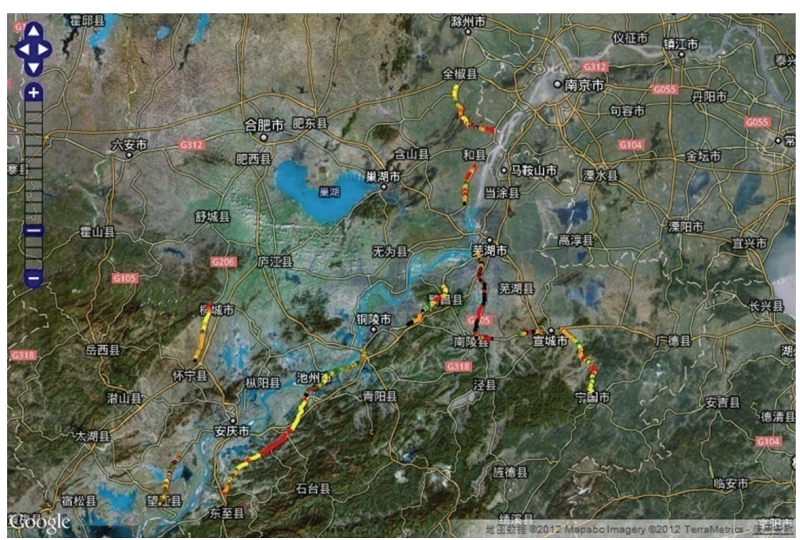

FIGURE 2: RIOH predicted accident rate decreasing map.

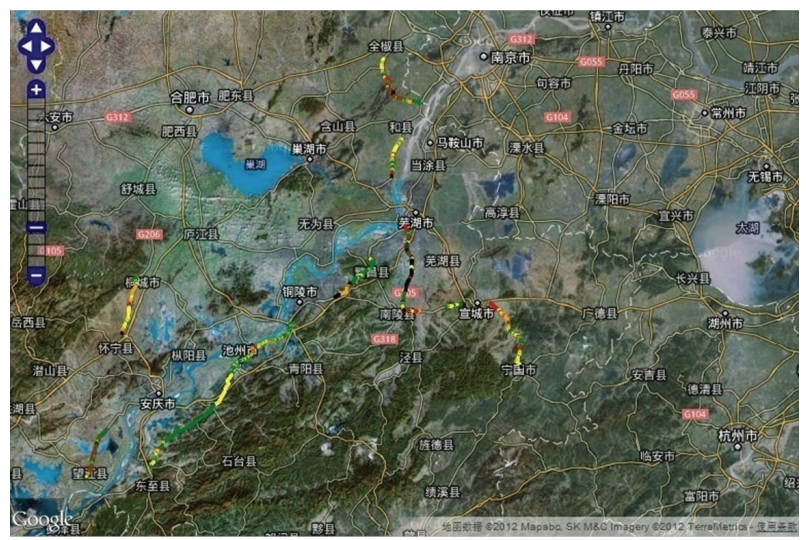

FIGURE 3: RIOH all occupants of risk rating map with countermeasures performance.

(Table 9). Levels 3, 4, and 5 sections are where the fewest could be prevented.

The percentage summary of future injury accidents decreasing levels if countermeasures are performed is shown in Table 10.

The Figure 3 map below shows the predicted risk rating map with countermeasures performance under the same standard in Table 11.

The predictive safety effects for all users if countermeasures are performed without changing traffic factors are 
TABLE 4: Key data and road attributes.

\begin{tabular}{|c|c|c|c|c|c|c|}
\hline \multirow{2}{*}{ Attribute } & \multirow{2}{*}{ Category } & \multicolumn{5}{|c|}{ Class } \\
\hline & & 1 & $2 \mathrm{a}$ & 2 & 3 & Urban \\
\hline Length $(\mathrm{km})$ & & 55.1 & 20.4 & 235.4 & 49.6 & 13.6 \\
\hline AADT & & 11305 & 9606 & 8013 & 3855 & 4006 \\
\hline \multirow{4}{*}{ Motorcycle flow (\% of AADT) } & $21 \%-40 \%$ & $0 \%$ & $0 \%$ & $0 \%$ & $100 \%$ & $0 \%$ \\
\hline & $11 \%-20 \%$ & $87 \%$ & $40 \%$ & $52 \%$ & $0 \%$ & $0 \%$ \\
\hline & $6 \%-10 \%$ & $13 \%$ & $60 \%$ & $36 \%$ & $0 \%$ & $0 \%$ \\
\hline & $1 \%-5 \%$ & $0 \%$ & $0 \%$ & $13 \%$ & $0 \%$ & $100 \%$ \\
\hline Bicycle flow & Low & $100 \%$ & $100 \%$ & $100 \%$ & $100 \%$ & $100 \%$ \\
\hline \multirow{4}{*}{ Pedestrian flow (along) } & High & $0 \%$ & $0 \%$ & $7 \%$ & $2 \%$ & $0 \%$ \\
\hline & Medium & $48 \%$ & $19 \%$ & $43 \%$ & $24 \%$ & $63 \%$ \\
\hline & Low & $49 \%$ & $77 \%$ & $46 \%$ & $74 \%$ & $37 \%$ \\
\hline & None & $3 \%$ & $4 \%$ & $3 \%$ & $0 \%$ & $1 \%$ \\
\hline \multirow{2}{*}{ Delineation } & Poor & $96 \%$ & $69 \%$ & $60 \%$ & $59 \%$ & $84 \%$ \\
\hline & Adequate & $4 \%$ & $31 \%$ & $40 \%$ & $41 \%$ & $16 \%$ \\
\hline \multirow{2}{*}{ Intersection quality } & Poor & $53 \%$ & $66 \%$ & $78 \%$ & $95 \%$ & $67 \%$ \\
\hline & Good & $47 \%$ & $34 \%$ & $22 \%$ & $5 \%$ & $33 \%$ \\
\hline \multirow{2}{*}{ Lane width } & Medium & $13 \%$ & $47 \%$ & $15 \%$ & $0 \%$ & $0 \%$ \\
\hline & Wide & $87 \%$ & $53 \%$ & $85 \%$ & $100 \%$ & $100 \%$ \\
\hline \multirow{3}{*}{ Curvature } & Sharp curve & $0 \%$ & $0 \%$ & $1 \%$ & $4 \%$ & $0 \%$ \\
\hline & Moderate curvature & $5 \%$ & $2 \%$ & $14 \%$ & $20 \%$ & $6 \%$ \\
\hline & Straight or gently curving & $95 \%$ & $98 \%$ & $86 \%$ & $76 \%$ & $94 \%$ \\
\hline \multirow{4}{*}{ Overtaking demand } & High & $0 \%$ & $0 \%$ & $83 \%$ & $0 \%$ & $0 \%$ \\
\hline & Medium & $0 \%$ & $0 \%$ & $0 \%$ & $99 \%$ & $0 \%$ \\
\hline & Low & $0 \%$ & $100 \%$ & $14 \%$ & $1 \%$ & $84 \%$ \\
\hline & None & $100 \%$ & $0 \%$ & $3 \%$ & $0 \%$ & $16 \%$ \\
\hline \multirow{3}{*}{ Side friction } & High & $0 \%$ & $0 \%$ & $0 \%$ & $1 \%$ & $1 \%$ \\
\hline & Medium & $2 \%$ & $3 \%$ & $2 \%$ & $0 \%$ & $1 \%$ \\
\hline & Low & $98 \%$ & $97 \%$ & $98 \%$ & $99 \%$ & $99 \%$ \\
\hline \multirow{3}{*}{ Pavement condition } & Poor & $0 \%$ & $0 \%$ & $0 \%$ & $53 \%$ & $0 \%$ \\
\hline & Medium & $4 \%$ & $1 \%$ & $5 \%$ & $29 \%$ & $0 \%$ \\
\hline & Good & $96 \%$ & $99 \%$ & $95 \%$ & $18 \%$ & $100 \%$ \\
\hline \multirow{9}{*}{ Roadside severity (right) } & Cliff & $0 \%$ & $0 \%$ & $1 \%$ & $0 \%$ & $0 \%$ \\
\hline & Motorcyclist friendly barrier & $1 \%$ & $0 \%$ & $0 \%$ & $0 \%$ & $1 \%$ \\
\hline & Distance to object $>10 \mathrm{~m}$ & $0 \%$ & $0 \%$ & $1 \%$ & $0 \%$ & $0 \%$ \\
\hline & Distance to object 5-10 m & $0 \%$ & $0 \%$ & $0 \%$ & $0 \%$ & $29 \%$ \\
\hline & Distance to object $0-5 \mathrm{~m}$ & $57 \%$ & $74 \%$ & $73 \%$ & $20 \%$ & $53 \%$ \\
\hline & Steep fill embankment slopes & $0 \%$ & $0 \%$ & $4 \%$ & $17 \%$ & $0 \%$ \\
\hline & Deep drainage ditches & $38 \%$ & $24 \%$ & $18 \%$ & $60 \%$ & $7 \%$ \\
\hline & Cut & $2 \%$ & $0 \%$ & $1 \%$ & $2 \%$ & $8 \%$ \\
\hline & Safety barrier & $1 \%$ & $2 \%$ & $3 \%$ & $1 \%$ & $1 \%$ \\
\hline \multirow{2}{*}{ Pedestrian crossing quality } & Poor & $62 \%$ & $39 \%$ & $39 \%$ & $100 \%$ & $36 \%$ \\
\hline & Adequate & $38 \%$ & $61 \%$ & $61 \%$ & $0 \%$ & $64 \%$ \\
\hline \multirow{3}{*}{ Speed } & $80 \mathrm{~km} / \mathrm{h}$ & $60 \%$ & $44 \%$ & $29 \%$ & $0 \%$ & $0 \%$ \\
\hline & $70 \mathrm{~km} / \mathrm{h}$ & $3 \%$ & $53 \%$ & $32 \%$ & $98 \%$ & $90 \%$ \\
\hline & $60 \mathrm{~km} / \mathrm{h}$ & $37 \%$ & $2 \%$ & $40 \%$ & $2 \%$ & $10 \%$ \\
\hline
\end{tabular}


TABle 5: Accident density (injury accident per km per year).

\begin{tabular}{|c|c|c|c|c|}
\hline Road number & General segments & Village segments & Intersection & All \\
\hline Road 1 & 0.67 & 0.72 & 0.14 & 0.51 \\
\hline Road 2 & 1.39 & 1.81 & 0.07 & 1.09 \\
\hline Road 3 & 0.84 & 0.39 & 0.03 & 0.42 \\
\hline Road 4 & 0.52 & 0.61 & 0.03 & 0.39 \\
\hline Road 5 & 0.35 & 0.14 & 0.01 & 0.16 \\
\hline Road 6 & 0.48 & 0.47 & 0.03 & 0.33 \\
\hline Road 7 & 0.89 & 0.63 & 0.03 & 0.52 \\
\hline Road 8 & 0.54 & 0.51 & 0.11 & 0.39 \\
\hline Road 9 & 0.00 & 0.64 & 0.05 & 0.23 \\
\hline
\end{tabular}

TABLE 6: Safety risk level summary results.

\begin{tabular}{lccccccccc}
\hline Level & & & \multicolumn{3}{c}{ Percentage (\%) } \\
& Road 1 & Road 2 & Road 3 & Road 4 & Road 5 & Road 6 & Road 7 & Road 8 & Road 9 \\
\hline 5 & 10.46 & 6.54 & 4.94 & 5.52 & 34.90 & 22.97 & 24.91 & 7.68 \\
4 & 2.57 & 0.00 & 0.00 & 0.00 & 27.60 & 25.15 & 0.00 & 3.84 \\
3 & 12.35 & 0.00 & 49.18 & 43.68 & 37.00 & 16.00 & 13.00 & 68.69 & 0.00 \\
2 & 65.00 & 4.58 & 14.12 & 49.00 & 0.00 & 36.00 & 34.00 & 17.17 & 95.44 \\
1 & 10.00 & 89.00 & 32.00 & 2.00 & 0.00 & 0.00 & 28.00 & 3.00 \\
\hline
\end{tabular}

TABLE 7: Basic condition of countermeasures.

\begin{tabular}{lccc}
\hline Countermeasures & Unit & Cost (Yuan) & Life cycle \\
\hline Center marking & Per linear km & 7500 & 2 \\
Roadside marking & Per linear km & 6000 & 2 \\
Angle adjustment & Location & 200000 & 10 \\
Lighting & Per linear km & 660000 & 10 \\
Pedestrian crosswalk & Location & 960 & 2 \\
Roadside barriers & Per linear km & 400000 & 10 \\
\hline
\end{tabular}

TABLE 8: Investment plan summary.

\begin{tabular}{|c|c|c|c|c|c|c|}
\hline Countermeasures & $\begin{array}{l}\text { Length } \\
(\mathrm{km})\end{array}$ & $\begin{array}{l}\text { Accident number } \\
\text { decreased per year }\end{array}$ & $\begin{array}{c}\text { Accident benefit of } 10 \\
\text { years (Yuan) }\end{array}$ & $\begin{array}{c}\text { Cost per year per } \\
\text { km }\end{array}$ & $\begin{array}{l}\text { Total cost } \\
\text { (Yuan) }\end{array}$ & $B / C$ \\
\hline Center marking & 185.3 & 33.32 & 802138853.79 & 37500 & 6948750 & 115.44 \\
\hline Roadside marking & 234.4 & 13.43 & 323290641.76 & 30000 & 7032000 & 45.97 \\
\hline Angle adjustment & 33.6 & 17.36 & 417883207.71 & 200000 & 67200000 & 6.22 \\
\hline Lighting & 53.2 & 12.10 & 291371924.31 & 660000 & 35112000 & 8.30 \\
\hline Pedestrian crosswalk & 5.7 & 0.94 & 22611161.78 & 4800 & 273600 & 82.64 \\
\hline Roadside barriers & 34.2 & 9.19 & 221229629.94 & 800000 & 13680000 & 16.17 \\
\hline Total & & 86.34 & 2078525419.30 & 1792300 & 130246350 & 15.96 \\
\hline
\end{tabular}

TABLE 9: Standard of injury accidents decreasing levels.

\begin{tabular}{lcc}
\hline $\begin{array}{l}\text { Road } \\
\text { user }\end{array}$ & $\begin{array}{c}\text { Accident decreasing } \\
\text { level }\end{array}$ & $\begin{array}{c}\text { Standard scope } \\
\text { (injury accidents decrease per km) }\end{array}$ \\
\hline & 5 & $0.000-0.048$ \\
\multirow{2}{*}{ All } & 4 & $0.048-0.201$ \\
& 3 & $0.201-0.435$ \\
& 2 & $0.435-0.896$ \\
& 1 & $>0.896$ \\
\hline
\end{tabular}

shown in Table 12, which indicates that there will be more levels 4 and 5 roads. In these 9 roads, road 2 will get the biggest results to decrease the level 1 segments, and road 1 , road 2 , and road 9 will get evident results to reduce low level segments.

\section{Conclusions and Prospects}

China is facing huge traffic safety problems with the increase of roadway length, traffic volume, and complex traffic composition. As a systematic method to determine road safety 
TABLE 10: Summary of injury accidents decreasing levels in network.

\begin{tabular}{lccccccccc}
\hline \multirow{2}{*}{ Decreasing level } & & \multicolumn{3}{c}{ Percentage (\%) } \\
& Road 1 & Road 2 & Road 3 & Road 4 & Road 5 & Road 6 & Road 7 & Road 8 & Road 9 \\
\hline 5 & 9.43 & 3.05 & 4.94 & 15.40 & 17.71 & 29.50 & 23.47 & 7.68 & 7.49 \\
4 & 21.61 & 3.27 & 44.71 & 40.46 & 41.67 & 19.01 & 19.86 & 29.29 & 28.99 \\
3 & 30.19 & 9.37 & 16.24 & 21.84 & 14.00 & 26.00 & 15.00 & 15.96 & 49.19 \\
2 & 33.00 & 42.48 & 22.82 & 22.00 & 27.00 & 15.00 & 24.00 & 47.07 & 14.33 \\
1 & 5.00 & 42.00 & 11.00 & 1.00 & 0.00 & 10.00 & 18.00 & 0.00 & 0.00 \\
\hline
\end{tabular}

TABLE 11: Safety risk level summary results for all users with countermeasures performance.

\begin{tabular}{lccccccccc}
\hline \multirow{2}{*}{ Decreasing level } & & \multicolumn{3}{c}{ Percentage (\%) } \\
& Road 1 & Road 2 & Road 3 & Road 4 & Road 5 & Road 6 & Road 7 & Road 8 & Road 9 \\
\hline 5 & 35.33 & 29.85 & 18.82 & 27.82 & 83.33 & 47.13 & 61.73 & 81.21 & 16.61 \\
4 & 27.62 & 10.68 & 62.59 & 25.52 & 16.67 & 24.75 & 0.36 & 11.92 & 45.93 \\
3 & 15.95 & 3.27 & 4.24 & 24.37 & 0.00 & 21.00 & 9.00 & 4.85 & 31.92 \\
2 & 18.00 & 14.60 & 10.35 & 22.00 & 0.00 & 7.00 & 27.00 & 1.82 & 4.89 \\
1 & 3.00 & 42.00 & 4.00 & 0.00 & 0.00 & 0.00 & 2.00 & 0.00 & 1.00 \\
\hline
\end{tabular}

TABLE 12: Safety risk level difference results for all users with countermeasures performance.

\begin{tabular}{lccccccccc}
\hline \multirow{2}{*}{ Decreasing level } & & & \multicolumn{3}{c}{ Percentage (\%) } \\
& Road 1 & Road 2 & Road 3 & Road 4 & Road 5 & Road 6 & Road 7 & Road 8 & Road 9 \\
\hline 5 & 24.87 & 23.31 & 13.88 & 22.30 & 48.44 & 24.16 & 36.82 & 73.54 & 13.36 \\
4 & 25.04 & 10.68 & 62.59 & 25.52 & -10.94 & -0.40 & 0.36 & 8.08 & 45.93 \\
3 & 3.60 & 3.27 & -44.94 & -19.31 & -37.50 & 4.95 & -4.69 & -63.84 & 31.92 \\
2 & -47.34 & 10.02 & -3.76 & -26.67 & 0.00 & -28.71 & -6.50 & -15.35 & -90.55 \\
1 & -6.17 & -47.28 & -27.76 & -1.84 & 0.00 & 0.00 & -25.99 & -2.42 & -0.65 \\
\hline
\end{tabular}

treatments for the agencies to ensure the most cost-effective returns in crash and injury reductions, traffic safety risk assessment has been researched and used widely all over the world. To match the actual safety conditions in China, RIOH is building ChinaRAP cooperating with iRAP in the pilot projects. Both RIOH model and iRAP model are used to find the model adaption, and this paper shows the RIOH method in the research, including the brief introduction of the model framework, the model procedure, and some initial results.

Under the pilot application, it is considered that total assessment result for whole road network is similar to the actual accident. For the investment plan, center marking, pedestrian crosswalk, and roadside marking are found to have the highest benefit cost ratios, which can reduce the injury accident evidently, such as road 2; about $47.28 \%$ level 1 roads will be upgraded to higher safety level roads, especially road 1, road 2, and road 9 which will get evident results to reduce low level segments.

Initially, ChinaRAP can be put into application to produce useful and helpful safety projects in China. However, building ChinaRAP must be a hard and long time work aiming for actual application. For example, limited by the traffic safety research of countermeasure effects, especially beforeand-after analysis, it is still difficult to get actual risk scores for some safety factors, and we cannot simply use foreign results without test in Chinese condition. In future works, the assessment results will be compared directly to actual accident distribution characteristic and then the deeply test and the adjustment of risk scores. Also large amount of traffic safety data accumulation, especially accident data, should be increased in order to direct before-and-after analysis and get more reasonable results.

\section{Conflict of Interests}

The authors declare that there is no conflict of interests regarding the publication of this paper.

\section{Acknowledgment}

The project is supported by the NSF Program of China (no. 51308263).

\section{References}

[1] http://www.moc.gov.cn/zhuzhan/tongjigongbao/hangyenianjian/201009/t20100927_844402.html.

[2] The People's Republic of China Road Traffic Accident Statistics, 2012.

[3] D. W. Harwood, F. M. Council, E. Hauer, W. E. Hughes, and A. Vogt, "Prediction of the expected safety performance of rural two-lane highways," Tech. Rep. FHWA-RD-99-207, United 
States Federal Highway Administration (FHWA), McLean, Va, USA, 2000.

[4] iRAP Toolkeys, http://www.irap.net.

[5] A. Vogt and J. Bared, "Accident models for two-lane rural segments and intersections," Transportation Research Record, no. 1635 , pp. 18-29, 1998.

[6] J. Hausman, B. H. Hall, and Z. Griliches, "Econometric models for count data with an application to the patents-R \& D relationship," Econometrica, vol. 52, no. 4, pp. 909-938, 1984.

[7] E. Hauer, J. C. N. Ng, and J. Lovell, "Estimation of safety at signalized intersections," Transportation Research Record, no. 1185, pp. 48-61, 1988.

[8] K. Rumar, P. Elsenaar, B. Marie et al., Road Safety Manual Recommendations from the World Road Association, PIARC, 2004.

[9] V. Shankar, F. Mannering, and W. Barfield, "Effect of roadway geometrics and environmental factors on rural freeway accident frequencies," Accident Analysis and Prevention, vol. 27, no. 3, pp. 371-389, 1995.

[10] P. Daly, G. Smith, J. Metcalfe, and R. McInerney, "AUSRAP: the Australian road assessment program," in Proceedings of the 22nd ARRB Conference: Research into Practice, ARRB Group, Canberra, Australia, 2006.

[11] J. Shen, A. Rodriguez, A. Gan, and P. Brady, "Development and application of crash reduction factors: a state-of-the-practice survey of State Departments of Transportation," in Proceedings of the 83rd Annual Meeting of the Transportation Research Board, 2004.

[12] D. W. Harwood, K. M. Bauer, I. B. Potts et al., "Safety effectiveness of intersection left- and right-turn lanes," Tech. Rep. FHWA-RD-02-089, US Department of Transportation, Federal Highway Administration, McLean, Va, USA, 2002.

[13] R. Elvik and T. Vaa, Eds., The Handbook of Road Safety Measures, Elsevier, Amsterdam, The Netherlands, 2004.

[14] Bureau of Transport Economics (BTE), "The black spot program 1996-2002: an evaluation of the first three years," Tech. Rep. 104, Bureau of Transport Economics (BTE), Canberra, Australia, 2001. 


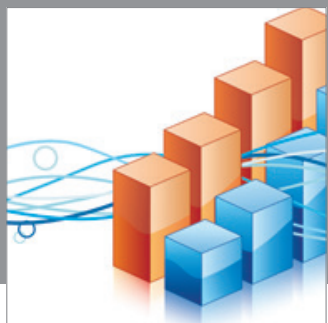

Advances in

Operations Research

mansans

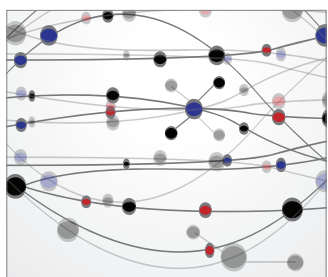

The Scientific World Journal
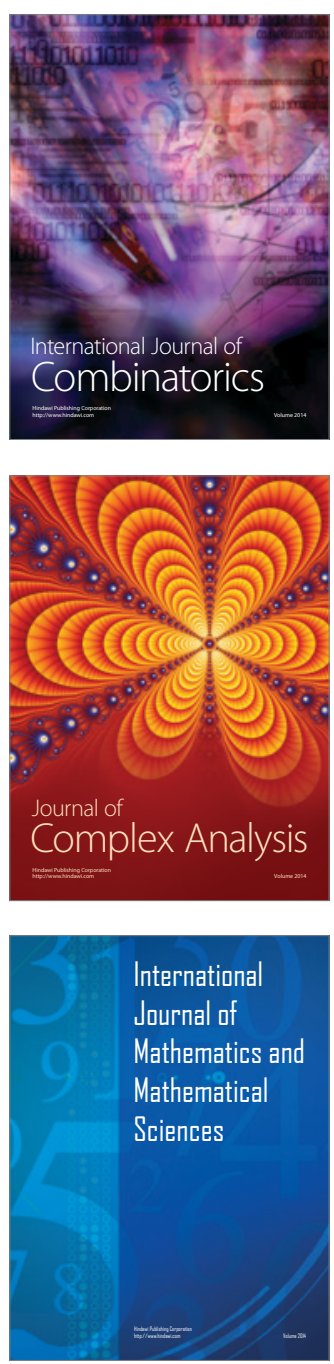
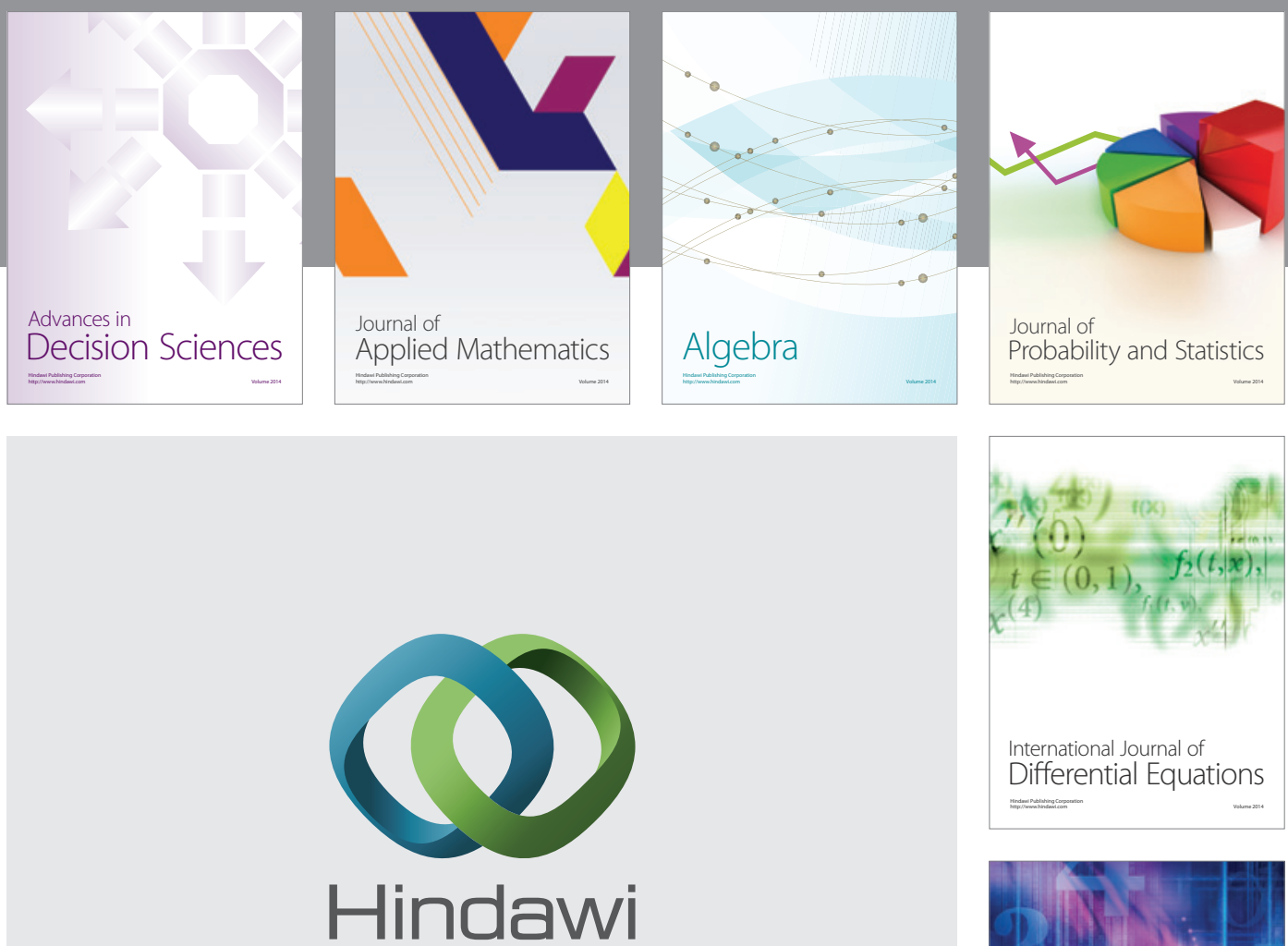

Submit your manuscripts at http://www.hindawi.com
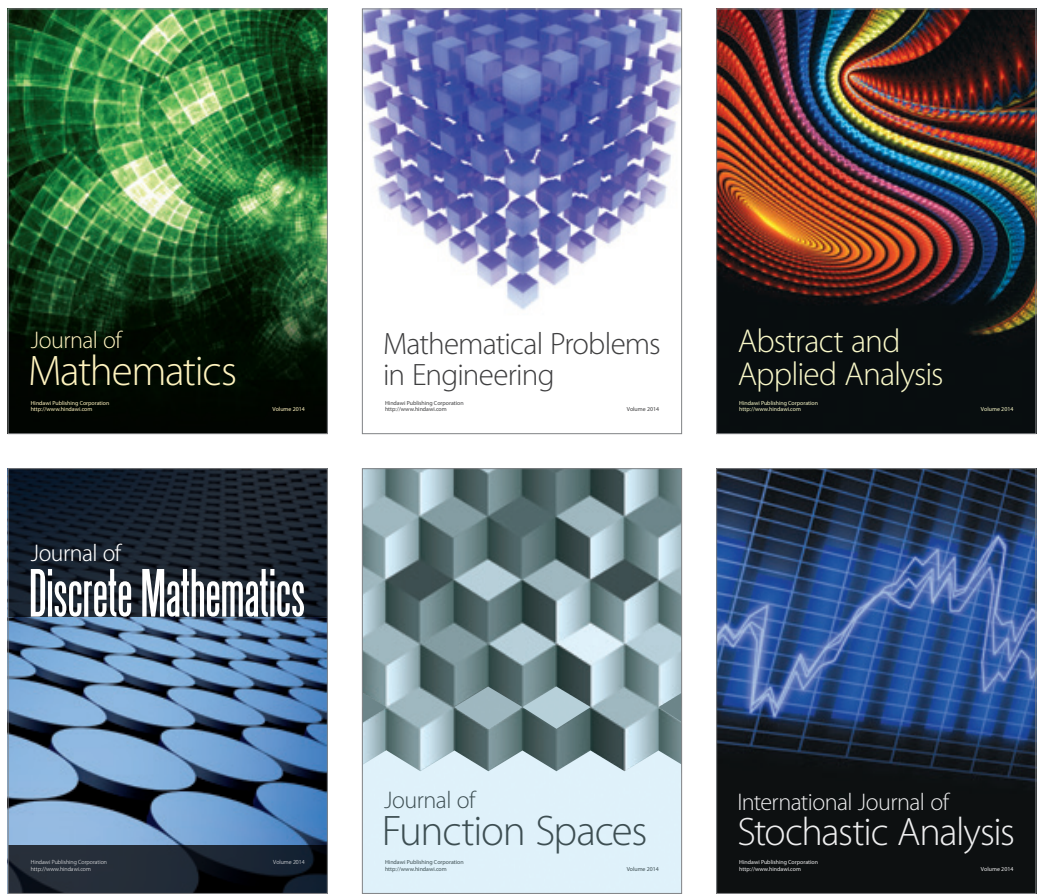

Journal of

Function Spaces

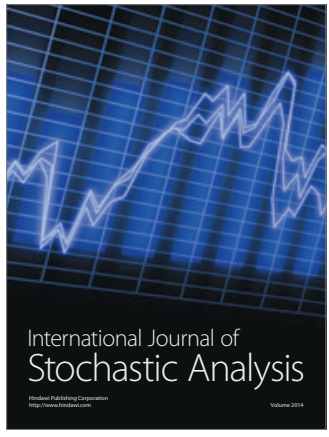

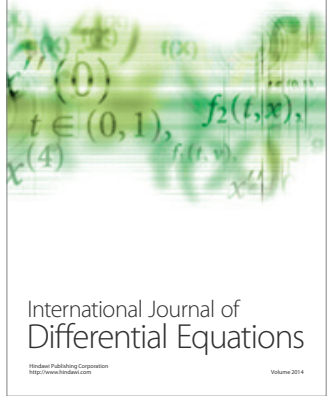
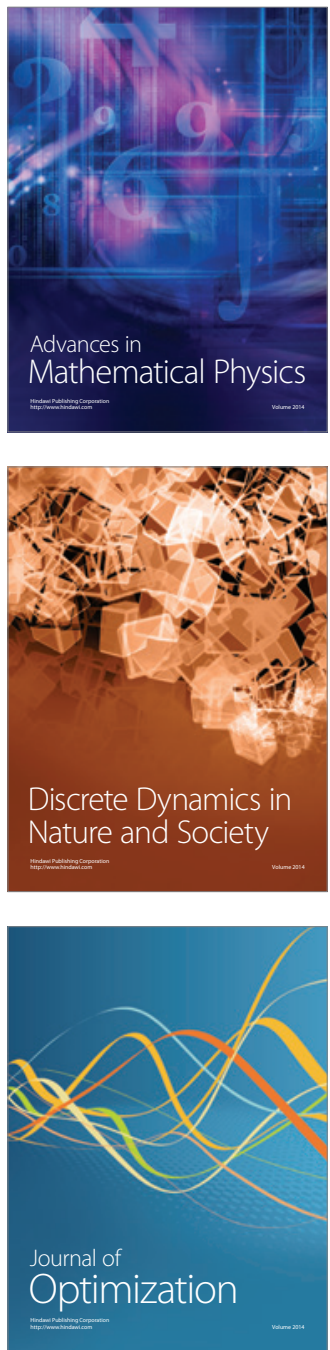